\title{
ROUNDUP360
}

Research

For other Roundups in this issue that cross-reference with Research see: Hip Roundup 3; Knee Roundups 1 and 5.

\section{Inflammation implicated in FAI}

- Femoroacetabular impingement (FAI) is one of the new diseases; the general orthopaedic consciousness leapt with joy as a potential explanation for the early onset osteoarthritis seen in the hips of some patients was mooted late last century. Since then, early intervention hip surgery has gone from strength to strength and there isn't a lump, bump or labral tear that an enterprising surgeon won't debride. However, the elephant in the room remains the missing link between mechanical impingement and subsequent degenerative change. If FAI doesn't in fact cause osteoarthritis, then the side-effect profile of the various interventions, either open or arthroscopic, might make them unattractive options in the longer term. The key may lie in what is happening at the level of impingement. Proving degeneration in the impinging cartilage would go some way to establishing a causal link. Researchers in Washington (USA) report this month on an ambitious study to do just that. The team harvested articular cartilage from control hips, those with FAl, and patients with various stages of osteoarthritis. Expression of inflammatory cytokines, chemokines and matrix regulatory proteins was measured using a qRT-PCR method and the level of gene expression compared between patients. The research team included 32 patients undergoing surgery for either hip preservation or undergoing total hip replacement. In addition, three control samples were also analysed. Intra-operative grading of the macroscopic appearances of the cartilage were noted and QRT-PCR undertaken to establish the relative gene expression. The results presented were quite clear. Although statistical significance is not normally sought in this type of biological study (due to the small numbers of cases available and the large numbers of analysis done, a ' $p$ ' value is essentially meaningless), these are powerful results. Of those biomarkers tested, only IL1-B and CXCL2 were unchanged between groups. The remaining chemokines (IL-8, CXCL1, CXCL2, CXCL6, CCL3 and $\mathrm{CCL}_{3} \mathrm{~L}_{1}$ ) were all raised in the impingement specimens, as were matrix degredating metalloproteinases and degredation products (collagen and aggrecan). The differences were significant for $\mathrm{CXCL}_{3}$, CXCL6 and COL2A1 versus the control group. There were a further four genes (IL-8, CCL3L1, ADAMTS-4 and ACAN) that were elevated in the impingement group versus both osteoarthritis and control genes. When compared with macroscopic findings, the gene expression was more marked in the middle stages of FAl (chondromalacia and cleavage/ thinning). The authors conclude that their results are consistent with a higher metabolic state during FAI, which supports the theory that FAI alters cartilage biology and that downstream degeneration may eventually take place. ${ }^{1}$ However, there are (like many similar studies) almost as many questions raised here as asked. For example, the observation that both ADAMTS-4 and $A C A N$ are raised in FAl is not unexpected with ADAMTS- 4 able to cleave aggrecan and ACAN encoding the molecule, suggesting a high turnover of aggrecan. However, it is surprising to find that these two markers are raised relative to the $\mathrm{OA}$ group as well as control tissue. We are some way off understanding the exact molecular mechanisms, but this study adds notably to our knowledge base.

\section{The whole package? Ponseti} and effective teaching

- The Ponseti method is the current standard of care for treatment of club foot in the majority of developed and developing countries. It offers an attractive treatment regime that can, for the most part, be undertaken without recourse to surgery more significant than an Achilles tendon release. The simple defined structure of the programme and the inexpensive nature of the equipment, combined with its reputation as the gold standard of care, have made Ponseti a priority for many charities and NGOs working in the developing world. While there is plenty of research outlining the effectiveness of an outreach Ponseti service in the developing world, surgeons from lowa City (USA), in combination with a comprehensive 'train the trainer' approach to implement the method, are studied in this paper. The study team used the introduction of the Ponseti method to Sri Lanka as a model for, and research evaluation of, the introduction of the Ponseti method in combination with a 'train the trainer' approach. They created an ethnographic study design that included interviews, focus groups, and direct observation of 162 patients and healthcare practitioners directly involved with clubfoot care. Patients at the time of the interview were around 18 months old and had travelled an average of $45 \mathrm{~km}$ to receive their health care. In the majority of cases, four casts were used (SD 2.2) for deformity correction. After initiation of the 'train the trainer' programme, demand for specialist clubfoot clinics grew tenfold (growing from six to seven patients per week to 60 per week). The majority of this was attributed to word of mouth. While cost of care was not a barrier to provision, there were some significant barriers to introduction of the technique, including lack of materials (casting and bracing) and poor provision for local anaesthetic tenotomy. ${ }^{2}$ The research team have effectively demonstrated that the 'train the trainer' method is an effective solution to the introduction of health care into this environment with rapid uptake. They do not present any long-term follow-up or clinical outcomes which are essential to establishing efficacy of this approach. 


\section{Unicompartmental knee design and tibial strain}

- Revision surgery for the unicompartmental knee is by nature of the design of the prosthesis an expected event. Patients can develop progression of the disease to other compartments, are generally younger and, as such are likely to require revision for aseptic loosening. However, despite this, between $25 \%$ and $40 \%$ of revisions are actually performed for pain. It is unclear why this should be the case, and one hypothesis is that the alteration in proximal tibial strain characteristics may be responsible at least in part for the pain. Researchers in Edinburgh (UK) set out to establish if the design of the prosthesis, both in terms of material and geometry, had a material effect on the predicted proximal tibial strain in cortical and cancellous bone models. Their study was conducted using composite SawBone proximal tibia, into which they implanted cemented unicompartmental knee components of differing designs (all polyethylene or metal-backed). They undertook a biomechanical study using stepped loading from $500 \mathrm{~N}$ to $2500 \mathrm{~N}$ in $500 \mathrm{~N}$ increments and the tibial strain was measured using a digital image correlation technique. Cancellous bone damage was estimated using sonic wave emission (a fancy technique for essentially recording the crunching of compression cancellous trabeculations). The cortical surfaces were remarkably similar in their perceived strain, with significant differences seen only at loads over $1500 \mathrm{~N}$ and only in the proximal $10 \mathrm{~mm}$. Acoustic emission demonstrated differences in cancellous bone damage at all loading points, with significant differences between the two implant types. The all-polyethylene implants were found to have significantly over 16 times the total number of cumulative acoustic emission hits as the controls and metal-backed group. These differences were also seen on unloading and in all likelihood represent a lack of implant stiffness. This was reflected in visible differences in macroscopic visible damage with the all-polyethylene tibias seeing much more visible damage than the other two groups, even at low loads. The authors conclude that "all-polyethylene implants should be used with caution in patients who are likely to impose large loads across their knee joint". ${ }^{3}$ While the conclusions of this study support the findings, it is important to remember that this is an unvalidated model and making clinical recommendations based on basic science studies is always tricky. We don't use all-polyethelene unicompartmental knee replacements but would stand a little bit shy of condemning those who do, based on a plastic bone and microphone study. We are sure the clinical results will support their findings in time.

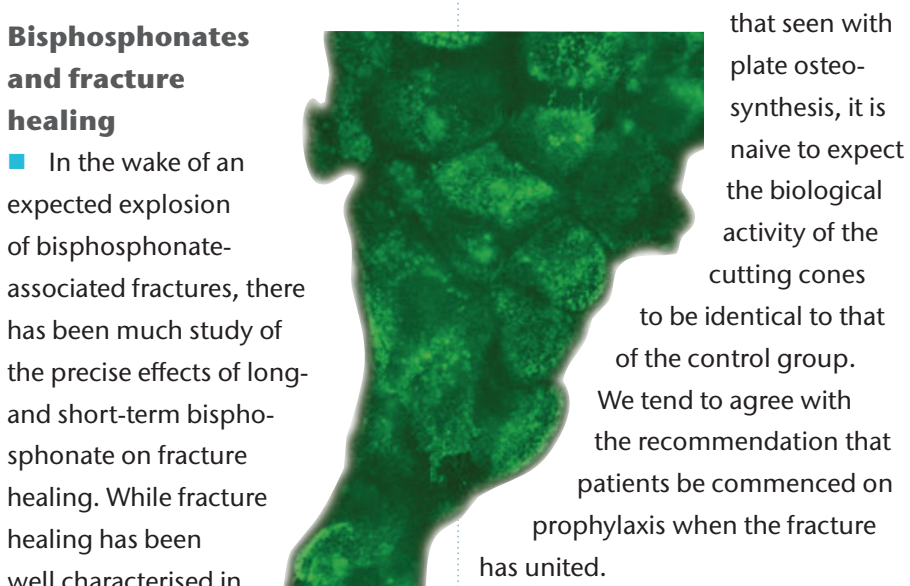
well characterised in experimental and animal studies in callus (biological) bone healing, there no data concerning the fracture direct healing and bisphosphonate use. Scientists in Newcastle (UK) used a validated rodent model of direct fracture healing. A standardised tibial osteotomy was performed and rigid compression plating employed. Their study used 20 skeletally mature SpragueDawley rats. The treatment group received daily subcutaneous injections of ibandronate $(1 \mu \mathrm{g} / \mathrm{kg})$ and the rats received saline for a period of three weeks. At the three-week point a controlled standardised tibial osteotomy was rigidly fixed with compression plating and the animals sacrificed at six weeks. Fracture repair was assessed with a combination of mechanical testing, radiographs and histology. The rats that received the ibandronate had a significantly lower mean stress to failure in four-point bending ( $8.69 \mathrm{Nmm}(-2)$ versus 24.65 $\mathrm{Nmm}(-2)$ ), and contact radiographs demonstrated a lower mean bone density at the osteotomy site than the controls ( $3.7 \mathrm{mmAl}$ versus 4.6 $\mathrm{mmAl}$ ). Histology demonstrated a similar picture with normal progression towards fracture union in the controls but impaired fracture healing in the bisphosphonate group with the persistence of cartilage-like and undifferentiated mesenchymal cells. ${ }^{4}$ The researchers have identified a significant finding. When reliant on cutting cone remodelling for fracture healing (direct bone healing) such as that seen with plate osteosynthesis, it is aive to expect to be identical to that of the control group. We tend to agree with the recommendation that patients be commenced on has united.

\section{Antibiosis in cement}

antibiotic-loaded cement has long been used as prophylaxis for infection in total joint replacement. The addition of antibiotics is not as simple as it might seem on the surface, with the inclusion of the antibiotic molecule in the polymer potentially affecting the biomechanical properties, the heat of polymerisation affecting the antibiotic and the potential for both to affect the elution characteristics of the antibiotic. As bacterial resistance profiles have changed, so have the range of antibiotics routinely included in the cement. Researchers in Taoyuan (Taiwan) have revisited the topic of antibiotic-loaded cements in view of the high rates of a contemporary array of bacteria including methicillin-sensitive Staphylococcus aureus (MSSA), methicillin-resistant Staphylococcus aureus (MRSA), coagulase-negative staphylococci (CoNS), Escherichia coli (E.coli), Pseudomonas aeruginosa ( $P$. aeruginosa), and Klebsiella pneumoniae. The researchers investigated the efficacy of standardised cement specimens made from $40 \mathrm{~g}$ PMMA cement loaded with $1 \mathrm{~g}$ antibiotics. The cement samples were investigated for elution characteristics, antibacterial activities, and compressive strength in vitro, covering all aspects of cement properties. The investigators identified that gentamycin had the longest elution time, while imipenem has an adverse effect on compressive strength (significant enough that the authors felt it rendered the cement unsuitable for prosthesis fixation) All antibiotics were able to maintain their antibacterial properties but given that gentamycin-loaded cement provided both broad antibacterial spectrum against all the test organisms and further had the greatest duration of antibacterial activity against MSSA, CoNS, P. aeruginosa and $E$. coli, the authors recommend continuing use of gentamycin as a standard of care. We agree. ${ }^{5}$

Zoledronic acid improves primary stability in revision?

- In contrast with the previous paper suggesting that bisphosphonates may be disadvantageous in fracture healing, researchers in Aarhus (Denmark) have investigated the potential for Zoledronic acid to aid achievement of primary stability in revision replacement. Noting that bulk allograft is often limited by resorption of the graft and loss of primary bony stability before the implants have osseointegrated, the research team theorised that as Zoledronic acid reduces bone turnover, its use in bulk allografting may delay or inhibit the resorption often seen with this kind of graft. To test the theory they hypothesised that zoledronate-impregnated allograft 
impacted around revision implants would improve implant fixation. They designed an animal model study to test this hypothesis and used mechanical push-out testing and histomorphometry as a surrogate marker for implant fixation. The study involved the use of 12 dogs, all of whom had a bone defect generated through implantation of 24 axially pistoning micromotion devices into the dogs' knees, generating a standardised revision model (including cavity, loose implant, fibrous tissue, and a sclerotic bone rim). Revision surgery undertaken two months later was with titanium revision components and impacted allograft soaked in either $0.005 \mathrm{mg} /$ $\mathrm{mL}$ zoledronate or saline and then impacted around the prosthesis. The study (zoledronate) group demonstrated a $30 \%$ increase in ultimate shear strength, a $54 \%$ increase in apparent shear stiffness and a $12 \%$ increase in total energy absorption, all of which were significant. At retrieval there was a threefold volume of allograft in the treatment but a similar volume fraction of new bone in the zoledronate group. ${ }^{6}$ The results from this study do suggest that a pretreatment with zoledronate is likely to be beneficial for the early stability of grafted revision replacement implants without any adverse effect on bone formation. We would agree with the authors that clinical studies are warranted.

\section{Osteoporotic fractures revisited?}

- The 'Bone and Joint decade' promoted by the World Health Organization has done an extremely good job of highlighting the plight of the many millions of patients worldwide suffering from fragility fracture and pushing their needs to the fore of research and clinical agendas. While clinical and scientific knowledge of low bone mineral density (BMD) fractures has soared, there is little known about the proportion of patients suffering fragility fractures with normal or near-normal bone mineral density. Researchers in Lexington (USA) took the opportunity to investigate a cohort of patients in a cross-sectional study with different patterns of fragility fractures. The research team identified three cohorts of patients; 25 with low energy fractures and normal BMD, 18 with low energy fractures and osteoporotic BMD, and 14 healthy volunteers to act as controls. Iliac crest bone samples were taken from each subject and tested using Fourier transform infrared spectroscopy and histomorphometry. The quality of the skeletal bone was assessed through use of dual x-ray absorptiometry and histomorphometry. The chief finding of this study was a difference in the collagen crosslinking ratio between the fracture groups and between the controls (13\% and $14 \%$, respectively). The cancellous bone volume was $29 \%$ greater and trabecular separation was $31 \%$ lower in the normal BMD subjects versus the low-BMD subjects with fractures. Bone turnover did not appear to differ between the groups, and there was no osteomalacia seen in this study. Patients with fragility fractures and normal BMD have been shown to maintain bone quantity, but the collagen crosslinking ratio was abnormal (a measure of bone quality).? This important human study identifies a group of patients with fragility fractures and collagen crosslinking abnormalities rather than bone mineralisation disorders. Other reports have indicated that altered collagen crosslinking is one factor associated with low fracture resistance. As our understanding of bone biology increases and how and why qualitative, as well as quantitative, disorders of bone are responsible for increased fracture risk, there is an increased chance of development and use of new treatments suitable to manage the underlying disorders.

\section{Electroarthrography for monitoring of cartilage degeneration}

- One of the challenges facing investigators designing studies to establish the efficacy, or otherwise, of cartilage sparing interventions and medicines is the difficulty associated with objective quantification of cartilage degeneration and ongoing, non-invasive monitoring over time. Researchers in Montréal (Canada) have developed and validated a new, simple technique called electroarthrography (EAG), which uses measured electrical potentials on the surface of the knee during joint loading. The study team undertook EAG recordings on 20 asymptomatic subjects and 20 patients with knee osteoarthritis (OA) who had had a unilateral TKR. An eight-electrodesites technique was used and measurements made as patients shifted their weight from one leg to the other to achieve joint loading. EAG repeatability was assessed with a test-retest protocol. This identified four electrode sites near the joint line as having the highest intra-class coefficients and mean EAG values.

There were no measurable potentials in the TKR and no significant correlation was found between amplitude and age, weight, height or body mass index. ${ }^{8}$ In what is a simple but useful paper, the authors have identified that EAG signals are likely to come from the streaming potentials in compressed articular cartilage. EAG is a promising technique that might be useful as a non-invasive assessment of cartilage degeneration and arthritis.

\section{REFERENCES}

1. Hashimoto S, Rai MF, Gill CS, et al. Molecular characterization of articular cartilage from young adults with femoroacetabular impingement. J Bone Joint Surg [Am] 2013;95A:1457-64.

2. Jayawardena $A$, Wijayasinghe $S R$, Tennakoon D, Cook T, Morcuende JA. Early effects of a 'train the trainer' approach to Ponseti method dissemination: a case study of Sri Lanka. lowa Orthop J 2013;33:153-60.

3. Scott CE, Eaton MJ, Nutton RW, et al. Proximal tibial strain in medial unicompartmental knee replacements: a biomechanical study of implant design. Bone Joint J 2013;95-B:1339-47.

4. Savaridas T, Wallace RJ, Salter DM,

Simpson AH. Do bisphosphonates inhibit direct fracture healing?: a laboratory investigation using an animal model. Bone Joint J 2013;95-B:1263-8.

5. Chang Y, Tai CL, Hsieh PH, Ueng SW. Gentamicin in bone cement: a potentially more effective prophylactic measure of infection in joint arthroplasty. Bone Joint Res 2013;2:220-6.

6. Sørensen M, Barckman J, Bechtold JE, Søballe K, Baas J. Preclinical evaluation of zoledronate to maintain bone allograft and improve implant fixation in revision joint replacement. $J$ Bone Joint Surg [Am] 2013;95-A:1862-8.

7. Malluche HH, Porter DS, Mawad H, Monier-Faugere MC, Pienkowski D. Lowenergy fractures without low $t$-scores characteristic of osteoporosis: a possible bone matrix disorder. $J$ Bone Joint Surg [Am] 2013;95-A:1391-6.

8. Préville AM, Lavigne $P$, Buschmann MD, et al. Electroarthrography: a novel method to assess articular cartilage and diagnose osteoarthritis by non-invasive measurement of load-induced electrical potentials at the surface of the knee. Osteoarthritis Cartilage 2013;21:1731-7. 